\title{
El ultrasonido en la insuficiencia respiratoria aguda. A propósito de un caso
}

\author{
Ultrasound for acute respiratory failure. A case repore
}

\author{
Patricia Calvo MD. 1, ${ }^{*}$, Ana Tejedor MD. ${ }^{1}$, Carmen Deiros MD. ${ }^{1}$, Josep Masdeu MD ${ }^{1}$. \\ 1 Servicio de Anestesiología, Reanimación y terapéutica del dolor. Consorci Sanitari Integral. Barcelona. España.
}

Fecha de recepción: 09 de noviembre de 2021 / Fecha de aceptación: 22 de noviembre de 2021

\section{Estimada Editora:}

a insuficiencia respiratoria aguda (IRA) es una de las patologías más habituales en la práctica clínica que mayor pericia exige para alcanzar un diagnóstico etiológico. Según datos europeos, la incidencia de IRA se estima de 77,6 y 88,6 casos por cada 100.000 habitantes/año, con una mortalidad hospitalaria asociada que oscila entre 9,8\%-49,4\%, dependiendo de la causa[14]. Una de las causas de IRA es el edema agudo de pulmón (EAP), que puede presentarse tanto de manera larvada como en pocas horas y cuya mortalidad en el paciente crítico varía entre el 32\%-58\%[1],[13]. En este contexto, la incorporación de herramientas clínicas que nos permitan un diagnóstico rápido y precoz y un posterior tratamiento dirigido, contribuyen sin duda a mejorar el pronóstico de nuestros pacientes.

Presentamos el caso de un varón de 53 años, con antecedentes de enolismo crónico severo activo, cirrosis hepática escala Child-Pugh B, varices esofágicas, pancreatitis crónica y diabetes mellitus insulino-dependiente, que fue intervenido de un absceso perianal sin incidencias intraoperatorias. En la unidad de Reanimación debutó con un cuadro progresivo de deterioro hemodinámico con hipotensión arterial y lactacidemia (7,2 mol/L). Se realizó una tomografía axial computerizada (TAC) abdominal que mostró una mínima lámina de líquido libre adyacente al reborde inferior del lóbulo hepático derecho, en fosa ilíaca derecha y región hipogástrica, sin otros hallazgos. Se orientó como shock séptico en contexto de absceso perianal. Se inició resucitación con fluidoterapia, soporte vasoactivo (SVA) y antibióticoterapia de amplio espectro. A las $48 \mathrm{~h}$ posoperatorias, tras una correcta evolución inicial con descenso progresivo del SVA y mejoría de los parámetros analíticos, el paciente presentó clínica de IRA con disnea, desaturación progresiva y crepitantes bibasales en auscultación. La gasometría arterial mostró una relación entre la presión parcial de oxígeno arterial y la fracción inspirada de oxígeno (PaFi: $\mathrm{PaO}_{2} / \mathrm{FiO}_{2}$ ) de $274 \mathrm{mmHg}$. Se realizó estudio ecográfico multisistémico a pie de cama observando a nivel pulmonar líneas B (> 3 por campo) sin lesiones inflamatorias en la línea pleural en ambos hemitórax; líneas A en campos superior y medio izquierdos y derrame pleural con atelectasia pasiva basal ipsilateral. La ecocardiografía transtorácica mostró una relación E/e' de 9, estimando una presión capilar pulmonar $(P C P)$ inferior a $18 \mathrm{mmHg}$, y unas resistencias vasculares sistémicas alrededor de 1.200 din-seg- $\mathrm{m}^{2} / \mathrm{cm}^{5}$. En la ecografía abdominal se visualizó una moderada cantidad de líquido ascítico. Analíticamente destacó hipoalbuminemia severa $(0,8$ $\mathrm{g} / \mathrm{dL}$ ). La clínica, los datos analíticos y ecográficos guiaron el diagnóstico a EAP[2], descartando razonablemente etiologías cardiaca[3] y séptica[4]. Fue orientado como cuadro de IRA por EAP no cardiogénico (EAPNC) de origen hipoalbuminémico en contexto de descompensación hepática. Se inició tratamiento con albúmina y furosemida, para forzar balances hídricos negativos, así como soporte ventilatorio no invasivo. Se realizó nueva TAC abdominal evidenciando aumento significativo de la cantidad de líquido libre en cavidad peritoneal de distribución difusa. Ante claudicación respiratoria, se procedió a intubación orotraqueal y ventilación mecánica invasiva. Pese al tratamiento precoz, el paciente acabó desarrollando fallo multiorgánico, siendo exitoso al cabo de pocos días.

La cirrosis es la fase final de la enfermedad hepática, dando lugar a una alteración homeostática generalizada. La pérdida de parénquima funcional provoca un aumento de las resistencias vasculares intrahepáticas, generando que el drenaje del territorio esplácnico al flujo sistémico sea a través de la circulación colateral, sin pasar por el hígado. Esto conduce a una depuración deficitaria de sustancias vasodilatadoras, como citoquinas, óxido nítrico, prostaglandinas (PG), sustancia p, entre otros. Dicha vasodilatación provoca una respuesta pobre frente a situaciones de bajo flujo, comprometiendo el aporte de $\mathrm{O}_{2}$ a los hepatocitos que sufren isquemia ante el estrés, agravando la disfunción del órgano, como sucedió a nuestro paciente. Paralelamente, el anabolismo proteico, predominantemente el de albúmina, que constituye el $85 \%$ de la presión coloidosmótica, suele ser insuficiente y en estos pacientes no se produce el incremento en la síntesis proteica, que puede llegar a ser del 200\%-300\% en contexto de estrés.

La disfunción hepática repercute sobre todo el organismo. A nivel respiratorio, la acumulación de sustancias vasoactivas

Patricia.CalvoPasaron@sanitatintegral.org

https://orcid.org/0000-0001-8262-5968 
no depuradas (PG, angiotensina, serotonina, etc), puede lesionar el endotelio vascular pulmonar y ser causa de EAP[10].

EI EAP lo clasificaremos en función de su fisiopatología o su etiología. Basándonos en la fisiopatología, encontramos el EAP hidrostático (producido por la diferencia entre gradientes de presión hidrostático y coloidosmótico de capilar e intersticio) y el EAP por lesión del endotelio capilar pulmonar, siendo su máxima expresión el síndrome de distrés respiratorio del adulto (SDRA). En función de la etiología, diferenciamos entre EAP cardiogénico (EAPC) y EAPNC. El fallo cardíaco y la sepsis son las causas más frecuentes de EAPC y EAPNC respectivamente. Se estima en 100.000 casos/año los pacientes ingresados por insuficiencia cardiaca aguda en España[13] y, de ellos, aproximadamente el $50 \%$ desarrolla un EAPC; por otra parte, la sepsis, con una incidencia anual de aproximadamente 20 casos por cada 100.000 habitantes, puede desembocar en un SDRA en un porcentaje variable según la serie consultada, alcanzando hasta $66 \%$ según Roca y cols[1]. No obstante, el papel de la hipoalbuminemia como causa de EAPNC es controvertido, si bien no se discute su papel como agravante del EAPNC[6] y su asociación a peores resultados[1],[2].

La ecografía cardiopulmonar está tomando un papel protagonista en el diagnóstico diferencial de EAPC y el EAPNC. En nuestro paciente, teniendo en cuenta las comorbilidades y los parámetros analíticos, fue clave para descartar el origen cardiogénico y séptico, llegar al diagnóstico de EAPNC de probable origen hepático y dirigir el tratamiento posterior. La presencia en la ecografía pulmonar de un patrón intersticial, esto es, 3 o más líneas B en un espacio intercostal con un patrón difuso, bilateral y homogéneo con línea pleural normal, indica acumulación de líquido en intersticio y permite realizar el diagnóstico de EAP; pero es la relación E/e' en ecocardiografía, que traduce las presiones de llenado en cavidades izquierdas, y que se correlaciona con la PCP, lo que descartó origen cardiogénico. Valores de E/e' < 15 van en contra del diagnóstico de EAP de origen cardiogénico[3]. El SDRA, normalmente cursa con signos de patrón intersticial difuso, bilateral, heterogéneo con afectación de la línea pleural, intercalándose alternativamente áreas sin afectación pulmonar con líneas B y, ocasionalmente, microconsolidados subpleurales.

Por último, la ecografía abdominal evidenció moderada cantidad de líquido libre abdominal, corroborado por TAC, que en el contexto del paciente nos guía hacia una posible descompensación ascítica en paciente con cirrosis hepática Child B previamente conocida. La confirmación analítica de hipoalbuminemia severa orienta hacia una pérdida de presión coloidosmótica por hipoalbuminemia en paciente cirrótico descompensado como causa de EAPNC, pudiendo iniciar, en consecuencia, además de tratamiento de soporte, tratamiento deplectivo y reposición de albúmina.

Concluimos que la IRA es una entidad relativamente frecuente que asocia una mortalidad elevada, sobretodo en el paciente crítico; con un diagnóstico diferencial tremendamente vasto que el anestesiólogo maneja en su práctica clínica habitual, haciendo que nuestro dominio de esta entidad sea especialmente importante. A raíz de este caso, valoramos los beneficios que ha reportado la ecografía como herramienta clínica en la orientación etiológica y la gravedad del cuadro, permitiendo con ello establecer un tratamiento; y la necesidad de incorporarla progresivamente en el campo del paciente crítico.

\section{Referencias}

1. Roca O, Sacanell J, Laborda C, Pérez M, Sabater J, Burgueño MJ, et al. Estudio de cohortes sobre incidencia de SDRA en pacientes ingresados en $\mathrm{UCI}$ y factores pronósticos de mortalidad. Med Intensiva. 2006 Jan-Feb;30(1):6-12. https://doi.org/10.1016/ S0210-5691(06)74455-2 PMID:16637425

2. Álvarez-Fernández JA, Núñez-Reiz A; en representación del Club de Ecografía UCI Madrid de la SOMIAMA. Clinical ultrasound in the ICU: changing a medical paradigm. Med Intensiva. 2016 May;40(4):246-9. https://doi.org/10.1016/j.medine.2015.10.003 PMID:26724248

3. Pérez del Villar C, Yotti R, Bermejo J. Técnicas de imagen en la insuficiencia cardiaca aguda. Rev Esp Cardiol. 2015;68(7):612-23. https://doi.org/10.1016/j.recesp.2015.02.021 PMID:26002273

4. Hirschhaut Schor E, Delgado Mosquera CJ, Cortes Montero MG. Ecografía pulmonar en cardiología: una ventana para el edema pulmonar. Rev Argent Cardiol. 2019;87(6):485-90. https://doi. org/10.7775/rac.es.v87.i6.16767.

5. Marteles MS, Urrutia A. Formas de presentación de la insuficiencia cardíaca aguda: edema agudo de pulmón y shock cardiogénico. Med Clin (Barc). 2014;142:14-9. https://doi.org/10.1016/ S0025-7753(14)70077-6.

6. Complicaciones de la fluidoterapia [Internet]. Uninet.edu. [citado el 6 de octubre de 2021]. Disponible en: https://www.uninet.edu/ tratado/c060208.html

7. Aguirre Sánchez MN. Hipoalbuminemia como factor predictor de mortalidad intrahospitalaria en cirrosis hepática descompensada Hospital II EsSalud Cajamarca perìodo 2010 - 2014. Universidad Privada Antenor Orrego - UPAO; 2015.

8. Mouret Hernández UEG, Mendoza Rodríguez M, López González A, Cortés Munguia A. Comparación de criterios de Berlín vs Kigali para diagnóstico del síndrome de insuficiencia respiratoria aguda. Med crít (Col Mex Med Crít). 2019;33(5):221-32

9. Gennaro D'Amico; Guadalupe Garcia-Tsao; Luigi Pagliaro (2006). Natural history and prognostic indicators of survival in cirrhosis: A systematic review of 118 studies. 44 (1), 0-231. https://doi. org/10.1016/j.jhep.2005.10.013.

10. Chávez-Tapia NC, Uribe M, López E. Complicaciones pulmonares de la cirrosis hepática: hipertensión portopulmonar y síndrome hepatopulmonar. La paradoja de la vasoconstricción y vasodilatación pulmonar [Internet]. Medigraphic.com. 2007 [citado el 8 de octubre de 2021]. Disponible en: https://www.medigraphic.com/ pdfs/gaceta/gm-2007/gm074h.pdf

11. Colmenero Ruiz M, Fernández Mondéjar E, Garcia Delgado M, Rojas M, Lozano L, Poyatos ME. Conceptos actuales en la fisiopatología, monitorización y resolución del edema pulmonar. Med Intensiva. 2006 Oct;30(7):322-30. https://doi.org/10.1016/ S0210-5691(06)74537-5 PMID:17067505

12. Arroyo V, Fernández J. Bases fisiopatológicas del uso de la albúmina humana en la cirrosis hepática. Gastroenterol Hepatol. 2012 Jan;35(1):42-9. https://doi.org/10.1016/j.gastrohep.2011.06.005 PMID:21944230

13. Rodríguez Mulero L, Carrillo Alcaraz A, Melgarejo Moreno A, Renedo Villarroya A, Párraga Ramírez M, Jara Pérez $\mathrm{P}$, et al. Factores de predicción del éxito de la ventilación no invasiva en el tratamiento del edema agudo de pulmón cardiogénico. Med Clin (Barc). 2005 Feb;124(4):126-31. https://doi. org/10.1157/13071006 PMID:15713241

14. Dueñas Castell C, Mejía Bermúdez J, Coronel C, Ortiz Ruiz G. Insuficiencia respiratoria aguda. Acta Colomb Cuid Intensivo. 2016;16:1-24. https://doi.org/10.1016/j.acci.2016.05.001. 